\title{
Phytochemicals, nutritionals and antioxidant properties of miracle fruit Synsepalum dulcificum
}

\begin{abstract}
Synsepalum dulcificum, also called the miracle fruit, which has the sweet-inducing activity can be used as additives in food, medicine and cosmetic industries. Some selected chemical properties of miracle fruit including percentage by weight, total anthocyanin, phenolic and antioxidant content of different parts of miracle fruit as well as physicochemical analysis of seed oil, nutritional elements of fruit juice were determined in this study. The results showed that miracle fruit contains a large amount of vitamin $\mathrm{C}(40.1 \mathrm{mg} / 100 \mathrm{~g}$ fresh fruit weight (FW)), phenolic content (625.57 mg GAE/100 g FW), high antioxidant capacity $(457.3 \mu \mathrm{mol}$ Trolox/100 g FW) and low total sugar content (5.6 g/100 g FW), suggesting that the fruit is healthy for human consumption. According to its fatty acid composition and Triacylglycerol (TAG) profile, miracle fruit seed oil is rich in oleic and palmitic acid.
\end{abstract}

Keyword: Synsepalum dulcificum; Physicochemical properties; Antioxidant; Seed oil; Triacylglycerol 